\title{
Patient quality of life and prognosis in multiple endocrine neoplasia type 2
}

\author{
Joanna Grey and Kym Winter \\ Association for Multiple Endocrine Neoplasia Disorders (AMEND), Tunbridge Wells, Kent, UK \\ Correspondence should be addressed to J Grey: jo.grey@amend.org.uk \\ This paper is part of a thematic review section on 25 Years of RET and MEN2. The guest editors for this section were Lois Mulligan and Frank Weber.
}

\begin{abstract}
Multiple endocrine neoplasia type 2 (MEN2) refers to the autosomal-dominant neuroendocrine tumour syndromes, MEN type 2A (MEN2A) and MEN type 2B (MEN2B). They are typified by the development of medullary thyroid cancer (MTC), phaeochromocytoma and parathyroid hyperplasia in MEN2A and MTC, phaeochromocytomas, ganglioneuromatosis and skeletal abnormalities in MEN2B. The aggressiveness of MTC is variable according to genotype, and although it is still the major cause of mortality in both conditions, prognosis has improved dramatically in those diagnosed and treated at a young age thanks to predictive genetic testing. Nevertheless, metastatic MTC, ganglioneuromatosis and a variety of other negative clinical and psychosocial impacts on quality of life and/or prognosis in MEN2 persist. In the absence, at the time of writing, of any large-scale research into quality of life specifically in MEN2, this review includes data from patient surveys and anonymised patient anecdotes from the records of the Association for Multiple Endocrine Neoplasia Disorders (AMEND), for whom the authors work. We recommend that these patients are cared for only in centres of expertise able to provide expert diagnosis, treatment and continuity of care, including psychological and transition support. Only in this way can the clinical advances of the last two and half decades be built upon further to ensure that the care of these complex, lifelong patients can be considered truly holistic.
\end{abstract}
Key Words
- gastrointestinal tract
- thyroid
- pheochromocytoma
- multiple endocrine neoplasias
- parathyroid

\section{Introduction}

Multiple endocrine neoplasia type 2 (MEN2) refers collectively to 2 main distinct types of autosomal dominantly inherited neuroendocrine tumour (NET) syndromes (Barakat et al. 2004); MEN type 2A and MEN type 2B (also known as MEN3). MEN2 predisposes patients to medullary thyroid cancer (99\%) and phaeochromocytoma (50\%) but also to other conditions according to specific genotype. MEN2A also includes a $25 \%$ risk of developing parathyroid hyperplasia and is now recognised as 4 separate syndromes: classic MEN2A,
MEN2A with cutaneous lichen amyloidosis (CLA), MEN2A with Hirschsprung's disease (HD) and familial MTC (Wells et al. 2015). MEN2B accounts for around 5\% of all MEN2 cases and also predisposes patients to diffuse intestinal ganglioneuromatosis and to mucosal neuromas and skeletal abnormalities. MEN2 is inherited in an autosomaldominant manner meaning that several generations in a single family may be affected by the same syndrome.

Medullary thyroid cancer (MTC) is the major cause of mortality in MEN2 and is more aggressive and presents 
earlier in MEN2B. However, the identification of the MTC biomarker, calcitonin, in 1968 and discovery of the causal RET (rearranged during transfection) proto-oncogene in 1993 (Donis-Keller et al. 1993, Mulligan et al. 1993) saw the emergence of prophylactic thyroid surgery based on mutation status, slashing mortality from MTC in MEN2 from 15 to 20\% down to around 5\% (Brandi et al. 2001). By 1996, the correlation between genotype and phenotype had become more defined (Eng et al. 1994, Mulligan et al. 1994) and International Consensus Guidelines were published in 2001 (Brandi et al. 2001). In 2006, tyrosine kinase inhibitor therapies emerged, providing hope for those with metastatic MTC. Nevertheless, timely and appropriate surgery remains the best chance of cure for MTC.

The risk of mortality from undiagnosed phaeochromocytoma is well documented (Prejbisz et al. 2013), and of particular significance in pregnancy. Patients continue to report difficulties with tumour diagnosis prior to RET oncogene mutation status diagnosis, suggesting persistent issues with early diagnosis in primary care settings. However, once diagnosed, prognosis from adrenalectomy has dramatically improved since the introduction of alpha-blockade to the pre-operative preparation. Improvements in surgical techniques over the last 25 years, moving from open to laparoscopic surgery when possible, have also minimised post-operative morbidity and hospital stay (Prejbisz et al. 2013), which may improve quality of life during recovery.

Despite all these advances, it is almost inevitable that diagnosis of a chronic complex syndrome like MEN2 will impact negatively on an individual's quality of life, and psychological wellbeing (Rodrigues et al. 2017). Combined with coping with diagnosis and acute and on-going clinical care, the inheritance aspect of MEN compounds a range of emotional issues that must be taken into account in the care of these patients. It is clear from Association for Multiple Endocrine Neoplasia Disorders (AMEND) Counselling Service sessions that there are a number of pre-existing factors that will either facilitate or impede an individual's capacity to cope with living with MEN2 over time, despite the condition's variable morbidity. An individual's ability to adapt well to living with the condition, to continue to find life meaningful and rewarding, and retain a measure of hopefulness and optimism will depend on a number of variables; temperament, social and financial circumstances, severity of symptoms, family dynamics, existential and personal meanings ascribed to being diagnosed with a genetic disorder and family dynamics, amongst others, all contribute to the individuals' experience of living with MEN2. In addition to highlighting clinical advances over the last 25 years, we attempt to give voice throughout to some of the on-going challenges and preoccupations faced by individuals living with MEN2.

\section{Methods}

Weidentified relevant articles using PubMed, supplemented with additional published materials. Additionally, in the absence, at the time of writing, of any large-scale research into quality of life specifically in MEN2, we lean heavily on data from the records of the AMEND, for whom the authors work. Data, anecdotes and anonymous vignettes were selected by the authors from AMEND membership and Research Registry application forms, patient experience surveys, submitted patient stories, Counselling Service data and notes from conversations with patients either individually or collectively at events held by the Association. The aim of these data is to provide a useful snapshot of the outstanding issues in quality of life as perceived by MEN2 patients themselves until such time as this area is studied more widely.

\section{Impacts on prognosis and quality of life in MEN2}

With such complex disorders, there are a variety of potential clinical impacts on both the prognosis and the quality of life of patients with MEN2, although some may be minimised with early diagnosis. These range from symptoms of tumour hormonal secretion to the balancing of post-operative replacement medications, such as levothyroxine and corticosteroids. The former may be exacerbated by delays in diagnosis, and the latter by patient compliance and prescribing gaps. Metastatic disease presents its own challenges in terms of tumour symptoms, treatment side effects and overall impact on emotional health.

MEN2 insinuates itself into all areas of familial, social, financial or psychological fragility, exploiting these preexisting vulnerabilities. The 2012 NHS Confederation Mental Health Network Report, 'Investing in emotional and psychological wellbeing for people with longterm conditions' (Mental Health Network and NHS Confederation 2012) states that the emotional and psychological needs of people with long-term conditions can be described through a continuum from healthy coping, through disease-related distress, to psychological and psychiatric conditions'. This is supported by the 
results of a small 2017 Brazilian study (Rodrigues et al. 2017) which looked at the correlation between resilience ('fighting spirit') and anxiety and depression in MEN2. While the majority of their cohort appeared to have lower mean scores for anxiety and depression, a notable $26 \%$ of the patients required regular psychotherapy. Negative psychological impact was seen particularly in those who were less informed about their disease and in those who were parents of children to whom they had passed on the condition. Throughout the activities of AMEND, we see similar themes emerging with respect to impacts on individual identity, fears for the future, work life and finances, relationships and on decisions about having children.

\section{Diagnosis}

As mentioned in the introduction, since the onset of RET gene testing, the number of deaths in MEN2 from MTC (the main cause of mortality) has dropped from 15-20\% down to around 5\% (Brandi et al. 2001). The prognosis for those diagnosed at an age that allows for appropriate prophylactic treatment $(<1$ year in MEN2B and commonly $<5$ years in MEN2A) and early instigation of regular monitoring is therefore greatly improved. Early thyroidectomies in children who have inherited MEN2 have been undertaken shortly after the MTC biomarker, calcitonin, was discovered in 1968. Without access to our current knowledge of genotypephenotype correlation, how many of these pre-gene test thyroidectomies were truly 'prophylactic' is open for debate. A recent study by the 'MEN2 in Children UK Collaborative Group' (unpublished at time of writing) (Nounou et al. 2017) looked at the outcomes of 79 MEN2 patients under 16 years of age between 1995 and 2013 who underwent thyroidectomy in the UK. Overall, prophylactic thyroidectomy in children with MEN2 in the UK was found to be safe. However, they conclude that surgical precision of prophylactic thyroidectomy can only be improved further if gene testing is performed as early as possible, leading to earlier thyroidectomy and reduced rates of lymph node dissections, thus potentially improving outcomes. Of course, this relies upon the early diagnosis of de novo or index case patients, something that remains challenging, particularly in primary care.

Phaeochromocytomas are catecholaminesecreting tumours of the adrenal glands which occur unilaterally or bilaterally in around $50 \%$ of the MEN2 patients. Their wide variety of symptoms, including episodic headaches, palpitations and sweating, can make phaeochromocytomas a challenge to diagnose and leave the sufferer at an increased risk of stroke or heart failure from hypertensive crisis. Indeed, 60\% of phaeochromocytomas during pregnancy remain undiagnosed, resulting in a $44 \%$ risk of miscarriage and $50 \%$ risk of maternal and foetal mortalities (Därr et al. 2012). An existing genetic diagnosis of MEN2 ensures the implementation of regular effective biochemical and radiological monitoring for these tumours to expedite early diagnosis and to improve prognosis.

Ganglioneuromatosis in MEN2B sits very much in the shadow of MTC when it comes to clinical research and yet, in those it affects, it can have serious impact on a patient's quality of life. A 2017 systematic literature review (Gfroerer et al. 2017) found that gastrointestinal symptoms, the most common being constipation (72.7\%), were reported in around half of all MEN2B patients within the first year of life, and yet the median age of diagnosis was 13 years.

Emily (33) is from the USA and was diagnosed with MEN2B at age 13. She had suffered with chronic constipation since birth and additionally with intermittent diarrhoea from age 19. '(The constipation) would build and build until I would get so backed up that I would throw up water, after which I would finally have diarrhoea for a day or so and get completely cleaned out. It was like clockwork: about 4 weeks of constipation followed by a day of diarrhoea and throwing up. I remember numerous times when I would drive an hour to work while throwing up'. Interestingly, Emily says that at each stage, her problems 'became the norm and I was used to it'. After contracting SIBO (small intestinal bacterial overgrowth) aged 28, things got significantly worse, but it was not until she was 32 that she underwent a colectomy, which revealed a twisted megacolon. A later ileostomy now presents its own issues: 'Even though sometimes my 'Baggie of Doom' and I argue, or it burns the ${ }^{[* * *]}$ out of me when I eat excessively spicy hot sauce, I would much rather experience its issues than ever go back to my colon issues'.

Overall, the average time to diagnosis in AMEND's patient population is 4 years (over 5 years in 26\%), reflecting other studies in rare diseases (Singh et al. 2016). In addition to the potential development of disabling symptoms from tumour hormone hyper-secretion and the ultimate mortality risks associated with this and with tumour metastasis, delays in diagnosis and initial misdiagnoses can also lead to patients losing trust and confidence in the medical profession. This may risk additional negative impacts on future care and disease management. 
(Jane, aged 48) 'I was diagnosed with MEN2A at age 30 after suffering for around 5 years with increasing symptoms from undiagnosed phaeochromocytomas. I was seen on multiple occasions by several GPs, a cardiologist and a psychiatrist over this time but constantly dismissed as mentally ill. I was at the point of giving up when a psychiatric hospital saved the day. It took a caring and experienced endocrine team to re-instil my sense of trust in the medical profession after my diagnosis, although I cannot help but retain a degree of mistrust in GPS to this day'.

The emotional impact of diagnosis depends upon a variety of factors, including the presence or absence of symptoms, other family members' experiences of the condition, the patient's age, cultural influences and attitudes and the capacity of healthcare professionals to break the news of the diagnosis appropriately, using sensitive language while allowing patients' space to understand and absorb information. In our experience, the majority of AMEND patient members diagnosed as a result of experiencing symptoms (often over a number of years) expressed relief at finally having a clear diagnosis and subsequent treatment and monitoring put in place. Coming to terms with the chronic and genetic natures of MEN2 seems to be somewhat easier for patients who had experienced symptoms pre-diagnosis, than for patients with few or no symptoms. The latter tend towards a greater degree of resentment, as opposed to relief, about the intrusion of testing and monitoring upon often already busy lives.

Teri (34) described being diagnosed with MEN2 in her 20s after her mother was diagnosed with the condition in her 50s. She said she 'didn't really think much about it' as she did not feel unwell. Living and travelling abroad for work, she 'shelved' the diagnosis, rebuffing her parent's attempts to raise the issue. Blood tests for fatigue that eventually led to evidence of phaeochromocytomas then caused a review of her whole understanding of the condition, and a confrontation with her own denial for which she was very angry, and self-condemnatory. AMEND Counselling saw her through the period of 're-diagnosis', bouts of anger and depression, anxiety over scanning and treatment procedures and a successful operation. Now in a new relationship, Teri is now considering the impact of her diagnosis upon her future decision making around having children.

For most patients, any initial impact of diagnosis seems to ameliorate over time, particularly if patients have the opportunity to work through the emotional impact and meaning of the diagnosis for them as an individual. Depression and anxiety are part of normal adjustments to the confusion and isolation of diagnosis with a rare genetic condition (Turner \& Kelly 2000), but with personal and/or professional support, most patients in our experience appear to adapt well over time to living with MEN2.

Around one-third of the patients experience guilt over passing on the disease to their children (Rodrigues et al. 2017). This manifests differently between families, but for some of our families, it can lead to considerable strain upon a couple's relationship, especially where the partnership had pre-existing vulnerabilities or tensions. Others found it strengthened family bonds, and others still that previous emotional issues between family members resurfaced. As Rob (38) put it, 'I've never thought I was like my father in any way - we never got on. I'm so angry the only thing he seems to have passed onto me is this'.

The generation of patients diagnosed with MEN2 by predictive gene testing in childhood are themselves now coming into young adulthood. For some, this raises uncommon pressures and complex ethical questions around their own decisions about starting a family: do they screen out the condition using pre-implantation genetic diagnosis, or not? One young woman (18) described that she felt, '...really strange about the idea of selecting my child; if someone had done that to me, I wouldn't have been born'.

\section{Treatment and living with MEN2}

Genotype-driven treatment and advancements in surgical techniques have undoubtedly helped to improve prognosis and quality of life in MEN2. Regular screening for phaeochromotycoma using urinary or plasma metanephrines and normetanephrines and MIBG scanning are particularly useful for early detection of these tumours in MEN2 patients, enabling timely intervention with lower associated morbidities (Därr et al. 2012). For instance, the introduction of laparoscopic transabdominal adrenalectomy in 1992, which has now been superseded by the retroperitoneoscopic approach, has decreased operating time, blood loss and complication rates. Combined with pre-operative preparation of patients with alpha-blockade to control hypertensive episodes and, where necessary, beta-blockers to control tachycardia, adrenal surgery is now the safest it has ever been.

However, numerous studies have shown that, in the hands of inexperienced or low-volume surgeons, complication rates rise in both thyroid cancer and adrenal surgery (Papaleontiou et al. 2017). Combined postthyroidectomy, hypocalcaemia rates for first time surgery as recorded by the British Association of Endocrine \& 
Thyroid Surgeons' (BAETS) UK Registry of Endocrine \& Thyroid Surgery were $23.6 \%$ (down from $29.6 \%$ in 2009) (Chawick et al. 2017), although it was acknowledged that a higher rate could be attributed to unavoidable removal or injury of the parathyroid glands during a central neck dissection at the time of thyroidectomy. BAETS consider that lower rates of post-thyroidectomy hypocalcaemia may be thought of as 'a surrogate marker of surgical performance'.

In MTC, the newest class of therapies (tyrosine kinase inhibitors), which aim to afford a modest but significant period of progression-free survival, come with varying side effects and subsequent impacts on quality of life, all of which are well documented (Wells et al. 2012, Elisei et al. 2013). Side effects of vandetanib and cabozanitinib include rash, nausea, fatigue, hand/foot syndrome and weight loss, most of which may be managed with additional medications, dose reductions or treatment 'holidays'. Indeed, these side effects may be seen by patients as a trade-off against progression-free survival and the hope for new treatments. Nevertheless, these side effects together with TKI resistance development, particularly in gene mutation codons 804 and 918 (Moserle et al. 2013), are driving a variety of current research efforts: optimisation studies into the current TKIs, vandetanib (NCT01945762 ClinicalTrials.gov) and cabozantinib (NCT01896479 ClinicalTrials.gov); the testing of newer compounds such as A80 (Plenker et al. 2017); phase II trials of multi-targeted TKI, lenvatinib (Schlumberger et al. 2016); the use of peptide receptor radionuclide therapy (PRRT) using radiolabelled somatostatin analogues (NCT01915485 ClinicalTrials.gov) and even immunotherapy (NCT03072160 ClinicalTrials.gov).

The emotional impact of pre-operative symptoms and post-operative effects upon quality of life can be extensive, with management of symptoms being a major feature and preoccupation causing anxiety and low mood/ depression in patients with MEN2 who contact AMEND's Counselling Service. A good example of post-operative effects is seen in primary adrenal insufficiency (AI) after bilateral adrenalectomy for phaeochromocytoma. With adrenal crisis still the cause of death in $15 \%$ of the patients with AI in the UK (Wass \& Arlt 2012), there is a real sense of nervousness for patients in returning home after surgery, often long distances by car from a specialist centre, without having been given an emergency injection kit and appropriate training that would be needed in the event of a road traffic accident during the journey. To address this, guidance from the Society for Endocrinology is clear on the need to provide an emergency kit and training for use, as well as educating patients about sick day rules in AI (Arlt 2016) but this may not always be reliably implemented throughout services.

Susan (48) has MEN2A and recently had her second adrenalectomy, resulting in adrenal insufficiency and therefore rendering her steroid dependent. Sue was discharged from hospital without an emergency injection kit or lessons in how to use one. After seeking advice from AMEND two weeks later, she requested a kit from her GP and was supplied with just one Solucortef kit, one needle and one syringe.

In our experience, fatigue is a commonly reported general issue, and one which can profoundly impact on a person's employment, education, parenting capacities and relationships as well as on their ability to manage their own emotional health. Patients who adapt to living optimally with MEN2 seem to be those who manage to achieve a certain 'flexibility of mind': focussing on the condition when necessary (e.g. around scans, medication, tests, increased symptoms) but otherwise allowing it to fade out of consciousness when the condition is well controlled. This 'resilience' is closely associated with better outcomes and quality of life for patients with chronic conditions (Sílvia et al. 2015).

Patients who are managing persistent, painful or distressing symptoms inevitably need the most support, and in such cases, working with them to explore what they can still comfortably manage, enjoy and take pleasure in is essential. It is notable that one-third of AMEND Counselling Service sessions between 2013 and 2016 involved patients disclosing suicidal thoughts that the majority felt unable to reveal to family, friends or health professionals due to feelings of shame and fear of negative consequences. In many families, the effect of living with MEN2 reveals a level of 'intergenerational trauma' around illness, bereavement and loss. Those with the condition often reveal family histories that cannot help but impact upon their capacity to be resilient, and perception of living with MEN2.

Polly (50) lost her father and several other relatives to MEN2A while growing up. 'For my generation, I think we have the psychological scars from the tragedy around us. I didn't think I would see 50 so I feel one of the lucky ones. There have been improvements in knowledge and patient information, treatment, knowing the genetics and counselling. I feel for those who are first generation, if we had been given different information, then we may have had a family but all we knew was death and the seeing the pain that caused'. 


\section{Support for the patient with MEN2}

\section{Psychological support services}

People with a long-term health condition are two to three times more likely to develop depression than the rest of the population (Department of Health 2011) and yet psychological services are not routinely represented within the MDT approach for MEN. Of the individuals who approached the AMEND Counselling Service, 12\% already had cognitive behavioural therapy for depression and/or anxiety. Many members found this helpful (especially in the initial relief of psychological symptoms) but others wanted more opportunity to explore further the meaning of their diagnosis in relation to its impact on their life choices, future, relationships and sense of self. Many clients expressed relief at being able to talk to a counsellor with knowledge of the condition rather than a generic cancer counselling service which can cause confusion over the status of their disease. The ability to access the service again in the future and avoid the need to repeat their medical story again was viewed positively.

For those few patients who may also have pre-existing psychiatric histories, concerns about how the two (or more) diagnoses interact can dominate. The diagnosis of an endocrine condition that can profoundly influence mood, and cause symptoms such as anxiety, caused some patients to review their entire understanding of their mental health history and difficulties. Interdisciplinary communication in such instances is essential, not least due to prescribing medications such anti-psychotic/antianxiolytic drugs and the potential contraindications of this. Anecdotally, patients with these two intersecting diagnoses do seem to struggle to find an interdisciplinary approach to their difficulties.

As Pat (45) put it, 'my psychiatrist doesn't seem interested in anything to do with my MEN, and my endocrinologist doesn't seem to take much account of my psychiatric history. And I can't make sense anymore of my anxiety, it's all so confusing. And also no one seems to be really taking into account about how all my medications might interact with each other, which really worries me'.

\section{Patient information and peer support}

It has been shown that a well-informed patient is likely to be a more resilient patient (Rodrigues et al. 2017). Historically, it was challenging for rare disease patients to find information on their disease written specifically for them and to benefit from peer support. The Internet age has completely transformed the information and support landscape and is helping to break the isolation that patients once felt so routinely. Whilst not all webbased information is accurate or suitable, and with some patient stories bound to terrify the newly diagnosed, the Internet also made the formation of professional patient advocacy groups and well-moderated online forums easier. With the support of a new generation of healthcare professionals keen to ensure that patients were cared for more holistically, these gaps in information and peer support are becoming a thing of the past. In fact, a 2016 meta-analysis (Allen et al. 2016) concluded that online support communities were useful in aiding selfmanagement, which in turn could improve a patient's experience with their long-term condition.

Nic (46) commented, 'just knowing there are other people, other families out there, living with it and getting on with their lives, been through what we're going through - that's been a huge relief since my daughter and I were diagnosed'.

\section{Hope}

The capacity to remain hopeful can have an enormously positive impact on all those suffering with chronic conditions, and has been found to be a key predictor of longer survival for both breast cancer patients and HIV sufferers (Turner \& Kelly 2000). It would be reasonable to predict that it has similar indications for MEN patients. Clinicians play an integral part in supporting their patients to be optimistic by their overall attitude to them as individuals, to the condition, and by engagement with new developments in the field. However, maintaining hopefulness should not be confused with 'positive thinking', which may involve denying or minimising the distress and limitations of the condition, or of its treatment. Patients consistently report feeling safer and more relieved when clinicians are able to discuss and attend seriously to the anxieties, fears and sadness that go with living with MEN without brushing them aside. Patients may already feel an expectation from family members, friends, society and their own 'inner voice' to think positively, and this can feel like an additional psychological burden at times. Thus, the skilful ability of a clinician to attend to a patient's fears, acknowledge real difficulties and remain hopeful can be invaluable.

\section{Other preoccupations of patients with MEN2}

These additional issues are often marginalised mentioned only briefly in articles, with very little additional information available on the impact on quality 
of life that these may incur within the syndromes. CLA and HD may occur in MEN2A, albeit rarely, and affect patients to varying degrees, with a corresponding impact on quality of life. For instance, some AMEND patient members have reported their CLA as a 'minor irritation', while others consider it a serious, sore and negatively impactful condition.

In societies that place heavy emphasis upon quite tightly proscribed notions of beauty and sexual attractiveness, MEN2B patients with the facial signifiers of the condition (mucosal neuromas and a distinct facial appearance) can experience particular emotional challenges. Several AMEND MEN2B members have described adolescence as a particularly difficult period, given its developmental preoccupations with appearance and sexual attractiveness both in person and in the media.

\section{Recommendations}

\section{Centres of expertise}

Patients with NETs who attend a Specialist Centre for their disease, such as a European Neuroendocrine Tumour Society (ENETS) certified Centre of Excellence, report feeling more optimistic, motivated and confident in their care, as well as more knowledgeable about their condition. They also feel less confused, frustrated or isolated than those who were not treated in a Specialist Centre (Singh et al. 2016). Too much uncertainty in engagement with the system and poor opportunities for communication between hospital appointments are often cited as major stressors for MEN patients and it is therefore essential that there is a concerted effort to concentrate MEN patients in centres of expertise. Within these, we recommend dedicated MEN outpatient clinics since these are seen by patients as acknowledging the condition as clinically important, thereby reinforcing a sense of security in their healthcare, reducing anxiety and possibly even increasing patient resilience. We would expect that Specialist Centres would be able to provide psychological support services, referral to trusted patient advocacy groups, an organised and supportive transition for adolescents from paediatric to adult care and key workers who can answer patients' out-of-clinic clinical queries or source answers in a timely manner. To help attend to the emotional aspects discussed in this review, additional training for clinical nurse specialists and physicians caring for patients with MEN2 on the psychological aspects of this disease would be helpful.

\section{More clinical and quality of life research into MEN2}

In the world of business, little happens without conducting market research to ensure that the right 'path to profit' is taken. Applying the same principle to the direction of research could be appropriate by way of an MEN2 needs analysis. At the WorldMEN meeting in 2016, a new approach was taken to this 2-yearly scientific meeting by including patients and patient advocates throughout the programme. The initiative, executed through close cooperation between the Dutch expert medical centre organisers and the Dutch patient group, Belangengroep MEN, included inviting direct feedback from participating patients into their perception of research needs. Fundamentally, this highlights the complete absence of large research studies into the quality of life in MEN2 (at time of writing). Such studies are much needed since these are likely to carry more weight in the medical community than any anecdotal evidence will.

It is encouraging that research into more effective treatments for MTC is continuing though opportunities remain for more interdisciplinary research to address those aspects of MEN2, such as ganglioneuromatosis, which have low mortality but a high impact on quality of life. Likewise, research into treatments for HD and CLA in MEN2A would be helpful. Hence, interdisciplinary research should extend not just between endocrinology, oncology and molecular biology, but might also encompass psychology/psychiatry, dermatology and gastroenterology, for example.

\section{Conclusion}

Clinically, there have been great improvements in diagnosis and treatment since the development of the gene test for MEN2, with age-appropriate, genotypeled prophylactic thyroid resection improving overall prognosis in patients diagnosed early in life. In addition, thyroid and adrenal surgery has never been safer, nor complication rates lower. Nevertheless, there is no room for complacency since these improvements in risk are still subject to diminishment when diagnosis is delayed and surgery is conducted outside of expert centres and by low-volume surgeons. Strong interdisciplinary communication would help to improve early diagnosis and prognosis further. A definitive cure for metastatic MTC remains elusive, although modest rates of progression-free survival are seen in TKI use, and on-going research provides hope for patients. Despite the clinical advances, there is still a considerable degree 
of negative impact on quality of life experienced by patients, not just from late diagnosis, tumour symptoms and metastatic disease, but also from psychosocial issues, not least when mental health problems pre-date the MEN2 diagnosis. Expert centres might ensure that multi-disciplinary teams include psychologists and/or undergo additional training in psychological aspects of genetic disease in order to help approach sensitively the diverse emotional impacts of MEN2. Patient peer support via routine referral to trusted patient groups should also be embedded in the service, together with a strong and supportive system for transition of adolescents from paediatric to adult care services. Overall, the future is becoming much brighter for families affected by MEN2, and by studying and addressing the outstanding clinical and psychosocial needs of patients, the outlook for future care and management could soon be truly holistic.

\section{Declarations of interest}

The authors declare that there is no conflict of interest that could be perceived as prejudicing the impartiality of this review.

\section{Funding}

This work did not receive any specific grant from any funding agency in the public, commercial or not-for-profit sector

\section{Acknowledgements}

The authors would like to thank all the patient members of the Association for Multiple Endocrine Neoplasia Disorders (AMEND) whose experiences were included in this review. AMEND has worked constantly since 2002 together with its expert medical advisors to provide information resources and support services to families in the UK and elsewhere affected by MEN disorders and related endocrine diseases.

\section{References}

Allen C, Vassilev I, Kennedy A \& Rogers A 2016 Long-term condition self-management support in online communities: a meta-synthesis of qualitative papers. Journal of Medical Internet Research 18 e61. (https://doi.org/10.2196/jmir.5260)

Arlt W \& Society for Endocrinology Clinical Committee 2016 SOCIETY FOR ENDOCRINOLOGY ENDOCRINE EMERGENCY GUIDANCE: Emergency management of acute adrenal insufficiency (adrenal crisis) in adult patients. Endocrine Connections 5 G1-G3. (https://doi. org/10.1530/EC-16-0054)

Barakat MT, Meeran K \& Bloom SR 2004 Neuroendocrine tumours. Endocrine-Related Cancer 11 1-18. (https://doi.org/10.1677/ erc.0.0110001)

Brandi ML, Gagel RF, Angeli A, Bilezikian JP, Beck-Peccoz P, Bordi C, Conte-Devolx B, Falchetti A, Gheri RG, Libroia A, et al. 2001 Guidelines for diagnosis and therapy of MEN type 1 and type 2 . Journal Clinical Endocrinology and Metabolism 86 5658-5671. (https://doi.org/10.1210/jcem.86.12.8070)
Chadwick D, Kinsman R \& Walton P 2017 Fifth national audit report of the British Association of Endocrine and Thyroid Surgeons. Henleyon-Thames, UK. (available at: http://www.baets.org.uk/audit/)

Därr R, Lenders JWM, Hofbauer LC, Naumann B, Bornstein SR \& Eisenhofer G 2012 Pheochromocytoma - update on disease management. Therapeutic Advances in Endocrinology and Metabolism 3 11-26.

Department of Health 2011 No health without mental health; a crossgovernment mental health outcomes strategy for people of all ages. London, UK: Department of Health.

Donis-Keller H, Dou S, Chi D, Carlson KM, Toshima K, Lairmore TC, Howe JR, Moley JF, Goodfellow P \& Wells SA Jr 1993 Mutations in the RET proto-oncogene are associated with MEN 2A and FMTC. Human Molecular Genetics 2 851-856. (https://doi.org/10.1093/hmg/2.7.851)

Elisei R, Schlumberger MJ, Müller SP, Schöffski P, Brose MS, Shah MH, Licitra L, Jarzab B, Medvedev V, Kreissl MC, et al. 2013 Cabonzantinib in progressive medullary thyroid cancer. Journal of Clinical Oncology 31 3629-3646. (https://doi.org/10.1200/ JCO.2012.48.4659)

Eng C, Smith DP, Mulligan LM, Nagai MA, Healey CS, Ponder MA, Gardner E, Scheumann GF, Jackson CE, Tunnacliffe A, et al. 1994 Point mutation within the tyrosine kinase domain of the RET protooncogene in multiple endocrine neoplasia type $2 \mathrm{~B}$ and related sporadic tumours. Human Molecular Genetics 3 237-241. (https://doi. org/10.1093/hmg/3.2.237)

Gfroerer S, Theilen TM, Fiegel H, Harter PN, Mittelbronn M \& Rolle U 2017 Identification of intestinal ganglioneuromatosis leads to early diagnosis of MEN2B: role of rectal biopsy. Journal of Pediatric Surgery 52 1161-1165. (https://doi.org/10.1016/j.jpedsurg.2016.10.054)

Mental Health Network and NHS Confederation 2012 Investing in emotional and psychological wellbeing for patients with long-term conditions. London, UK: NHS Confederation. (available at: www. nhsconfed.org)

Moserle L, Jiménez-Valerio G \& Casanovas O 2014 Antiangiogenic therapies: going beyond their limits. Cancer Discovery 4 31-41. (https://doi.org/10.1158/2159-8290.CD-13-0199)

Mulligan LM, Kwok JBJ, Healey CS, Elsdon MJ, Eng C, Gardner E, Love DR, Mole SE, Moore JK, Papi L, et al. 1993 Germ-line mutations of the RET proto-oncogene in multiple endocrine neoplasia type $2 \mathrm{~A}$. Nature 363 458-460. (https://doi.org/10.1038/363458a0)

Mulligan LM, Eng C, Healey CS, Clayton D, Kwok JB, Gardner E, Ponder MA, Frilling A, Jackson CE, Lehnert H, et al. 1994 Specific mutations of the RET proto-oncogene are related to disease phenotype in Men 2A and FMTC. Nature Genetics 6 70-74. (https://doi.org/10.1038/ng0194-70)

Nounou H, Prete F, Abdel-Aziz T, Morkane C, Brain C \& Kurzawinski TR 2017 Can precision medicine be done with surgical precision: prophylactic thyroidectomy in children with MEN2 in the United Kingdom. Presented at 7th Symposium of the European Society of Endocrine Surgeons: Surgery for Subclinical Endocrine Disease (April 6-8 2017, Oxford, UK). Langenbeck's Archives of Surgery (Supplement) abstract P97. (available at: https://slideheaven. com/7th-symposium-of-the-european-society-of-endocrinesurgeons-surgery-for-subclini.html)

Papaleontiou M, Hughes DT, Guo C, Banerjee M \& Haymart MR 2017 Population-based assessment of complications following surgery for thyroid cancer. Journal of Clinical Endocrinology and Metabolism 102 2543-2551.

Plenker D, Riedel M, Brägelmann J, Dammert MA, Chauhan R, Knowles PP, Lorenz C, Keul M, Bührmann M, Pagel O, et al. 2017 Drugging the catalytically inactive state of RET kinase in RETrearranged tumors. Science Translational Medicine 9 eaah6144. (https://doi.org/10.1126/scitranslmed.aah6144)

Prejbisz A, Lenders JW, Eisenhofer G \& Januszewicz A 2013 Mortality associated with phaeochromocytoma. Hormone and Metabolic Research 45 154-158. (https://doi.org/10.1055/s-0032-1331217) 
Rodrigues K, Toledo RA, Coutinho FL, Nunes AB, Maciel RMB, Hoff AO, Tavares MC, Toledo SPA \& Lourenço DM Jr 2017 Assessment of depression, anxiety, quality of life, and coping in long-standing multiple endocrine neoplasia type 2 patients. Thyroid 27 693-706. (https://doi.org/10.1089/thy.2016.0148)

Schlumberger M, Jarzab B, Cabanillas ME, Robinson B, Pacini F, Ball DW McCaffrey J, Newbold K, Allison R, Martins RG, et al. 2016 A phase II trial of the multitargeted tyrosine kinase inhibitor lenvatinib (E7080) in advanced medullary thyroid cancer. Clinical Cancer Research 22 44-53. (https://doi.org/10.1158/1078-0432.CCR-15-1127)

Sílvia Fernanda C, Ribeiro de Sá L, Eugênia Glustak M \& Santiago MB 2015 Resilience in chronic diseases: a systematic review. Journal of Cogent Psychology 2 article 1024928. (https://doi.org/10.1080/233119 08.2015.1024928)

Singh S, Granberg D, Wolin E, Warner R, Sissons M, Kolarova T, Goldstein G, Pavel M, Öberg K \& Leyden J 2016 Patient-reported burden of a Neuroendocrine Tumor (NET) diagnosis: results from the first global survey of patients with NETs. Journal of Global Oncology 3 43-53. (https://doi.org/10.1200/JGO.2015.002980)

Turner J \& Kelly B 2000 Emotional dimensions of chronic disease. Western Journal of Medicine 172 124-128. (https://doi.org/10.1136/ ewim.172.2.124)

Wass JAH \& Arlt W 2012 How to avoid precipitating an acute adrenal crisis. BMJ 345 e6333. (https://doi.org/10.1136/bmj.e6333)

Wells SA, Robinson BG, Gagel RF, Dralle H, Fagin JA, Santoro M, Baudin E, Elisei R, Jarzab B, Vasselli JR, et al. 2012 Vandetanib in patients with locally advanced or metastatic medullary thyroid cancer: a randomized, double-blind Phase III trial. Journal of Clinical Oncology 30 134-141. (https://doi.org/10.1200/JCO.2011.35.5040)

Wells SA Jr, Asa SL, Dralle H, Elisei R, Evans DB, Gagel RF, Lee N, Machens A, Moley JF, Pacini F, et al. 2015 Revised American Thyroid Association Guidelines for the management of medullary thyroid carcinoma. Thyroid 25 567-610. (https://doi.org/10.1089/ thy.2014.0335)

Received in final form 3 October 2017

Accepted 24 October 2017

Accepted Preprint published online 24 October 2017
(C) 2018 Society for Endocrinology Published by Bioscientifica Ltd. Printed in Great Britain 